\title{
The 23 July 2012 SEP event numerical simulation with multi-spacecraft observation data
}

\author{
S.-Y. Qi ${ }^{a, *}$ and G. Qin ${ }^{b}$ \\ ${ }^{a}$ College of Sciences, Henan Agricultural University, \\ Zhengzhou, China \\ ${ }^{b}$ School of Science, Harbin Institute of Technology, \\ Shenzhen, China \\ E-mail: qsyxiaoyi@163.com, qingang@hit.edu.cn
}

23 July 2012, multiple-spacecraft, namely STEREO-A, STEREO-B, and ACE, observed an extremely powerful, superfast interplanetary coronal mass ejection (ICME) together with the ICMEdriven shock and associated solar energetic particles (SEPs). We analyze the relationship between the propagation of the shock and the SEP flux with the Parker spiral magnetic field model. Moreover, we simulate the SEP event by numerically solving the three-dimensional focused transport equation of SEPs considering the shock as the moving source of energetic particles. We use the same diffusion model format for the simulations of protons and electrons but with different parameters for simplicity. The simulation results can qualitatively explain the important features of the SEP flux observed by the multiple spacecraft simultaneously. Additionally, the numerical results for both energetic protons and electrons approximately agree with multi-spacecraft observations.

$37^{\text {th }}$ International Cosmic Ray Conference (ICRC 2021)

July 12 th - 23rd, 2021

Online - Berlin, Germany

\footnotetext{
*Presenter
} 


\section{Introduction}

On 23 July 2012, a halo superfast ICME was launched from the Sun with a maximum speed reaching $3050 \pm 260 \mathrm{~km} \mathrm{~s}^{-1}$ with the magnetic field of the ejecta reaching $109 \pm 1 \mathrm{nT}$ [1]. Significant SEPs assumed to be accelerated by the ICME-driven interplanetary shock were detected during this event [2].

Here, we investigate the extreme 23 July 2012 SEP event observed by multi-spacecraft STEREO$A$, STEREO-B, and ACE. We apply a simple and ideal Parker spiral magnetic field model to analyze the magnetic connections of the shock to the multiple spacecraft with the outward propagating CME. Furthermore, we study SEP flux by numerical simulations using the parameters observed by the spacecraft, and we compare our results with the observations. We use the numerical Shock Particle Transport Code (SPTC) by [3] which considers an ICME shock to be a moving source of SEPs. In section 2, we present the observations and analysis. In section 3, we show the transport model. In section 4 , we show our simulations and the comparisons with observations. In section 5, we present the conclusion and discussion.

\section{Observations and analysis}

The 23 July 2012 CME was observed launching from the Sun by the EUVI observations of the SECCHI instrument onboard the two STEREO spacecraft at about $0208 \pm 2$ minutes UT [2]. The source location was the sunspot group NOAA 11520 at $\mathrm{S}^{\circ} 5^{\circ} \mathrm{W} 133^{\circ}$ [1]. The fast forward shock driven by the CME was observed to reach STEREO-A at 20:55:25 UT $\left(T_{S T A}\right)$. At 22:55 UT, the leading edge of the ICME reached STEREO-A in the ecliptic with latitude and longitude $\mathrm{S} 0.07^{\circ} \mathrm{W} 121.3^{\circ}$, and heliocentric distance 0.96 au [2]. The latitude and longitude of STEREO-B is $\mathrm{S} 0.16^{\circ} \mathrm{E} 115.2^{\circ}$. Later, the shock reached STEREO-B at 21:21:01 UT $\left(T_{S T B}\right)$, but there was no shock encounter on Advanced Composition Explorer (ACE) which was $121^{\circ}$ east of STEREO-A.

In order to determine the magnetic connections between the multiple spacecraft together with the shock front, we use a schematic (Figure 1) which shows their relative positions. We use the Parker spiral model to describe the magnetic field for simplicity. Figure 1 shows such a schematic in the ecliptic plane at 20:55 UT on 23 July, 2012. The red and blue spirals depict Parker spiral magnetic field lines passing through STEREO-A and STEREO-B at W121.3, $0.96 \mathrm{au}$, and E115.2, $1.02 \mathrm{au}$, respectively. The black spiral represents a Parker magnetic field line passing through $A C E$ at W0, 1 au. The black straight line points to the direction of the shock nose. The yellow area indicates the scope of the shock sweep with width $W_{s}$, the possible value of which can be deduced based on spacecraft observations.

The time profiles of proton fluxes observed by the multiple spacecraft during the 23 July 2012 SEP event are exhibited in Figure 2. The above proton channels with similar energy are chosen for comparison. In order to investigate the transport of the CME-driven shock and its contributions to the SEP fluxes detected by the multiple spacecraft, we divide the proton flux time profile into several parts by time with various vertical lines according to some key moments in the propagation of the shock. The dotted vertical line indicates $T 1,14: 08: 00$ UT on July 23, 2012, when the shock connection for $A C E$ was lost. The red and blue vertical solid lines indicate the shock arrival time, 20:55 UT at STEREO-A and 21:21 UT at STEREO-B, respectively, which almost coincide in Figure 


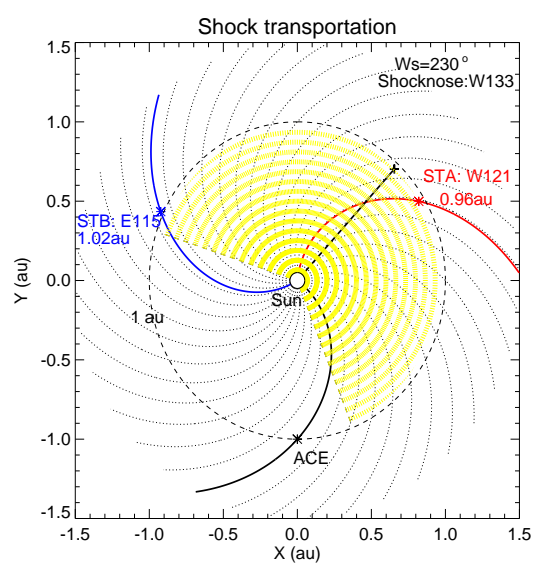

Figure 1: Positions of the spacecraft and the shock nose direction in the ecliptic plane at 20:55 UT on 23 July 2012, the time when the shock passed STEREO-A.

2. The dashed vertical lines indicate $T 2,15: 19: 59$ UT on July 25 , when the connection of $A C E$ was reestablished. In addition, the dot-dashed vertical lines indicate $T 3,06: 56: 00$ UT on July 27, when the connection of STEREO-B was lost again. Similarly to Figure 2, the electron flux time profile is shown in Figure 3.

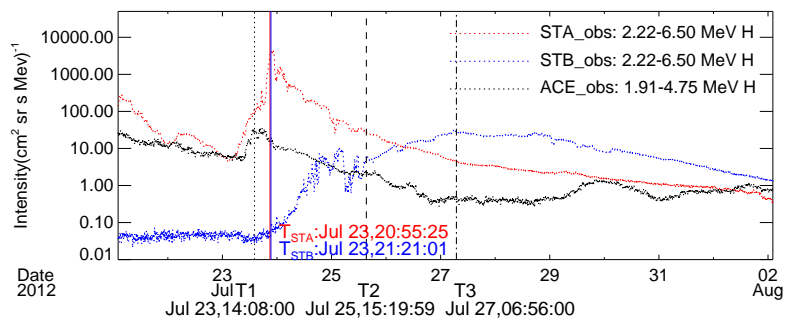

Figure 2: Time profile of proton fluxes by the multi-spacecraft observation during the 23 July 2012 SEP event.

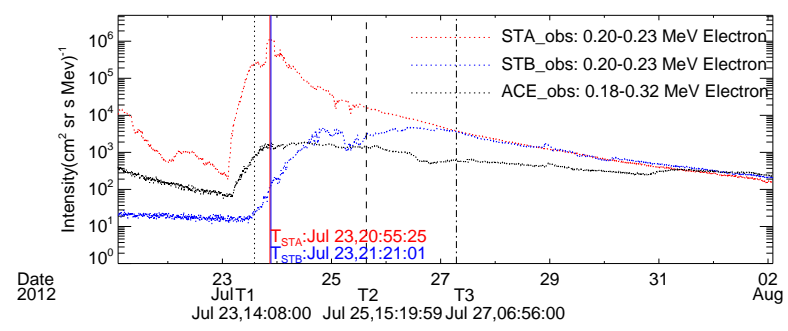

Figure 3: Time profile of electron fluxes by the multi-spacecraft observation during the 23 July 2012 SEP event.

At $T 1$, as the cobpoint of STEREO-A, which indicates the location of the shock front that is magnetically connected to the observer [3,5], is near the shock nose with the acceleration efficiency much higher than at the shock edge, the SEP flux detected by STEREO-A (the red curves in Figure 
2 for protons and Figure 3 for electrons) was much higher than the background level. In addition, when the shock reached STEREO-A at 0.96 au, $T_{S T A}$, the SEP flux of STEREO-A almost reached the peak. We note that during the shock crossing there was a significant increase of the SEP flux observations by STEREO-A. Soon after, shock reached STEREO-B at 1.02 au at $T_{S T B}$. Afterward, as the shock passed STEREO-A, the SEP flux detected by STEREO-A dropped dramatically.

Solar energetic particle flux detected by STEREO-B remained at background level before $T 1$ because the shock was not wide enough to cover STEREO-B's magnetic field line (Figure 4). However, as connection to the shock was established some time after $T 1$, the cobpoint of STEREO$B$ was moving toward the shock nose and the SEP flux observed by STEREO-B rose with time. We note that the SEP flux observation of STEREO-B began to rise around $T 1$ before the connection was actually established, one may assume that this is due to perpendicular diffusion of the energetic particles.

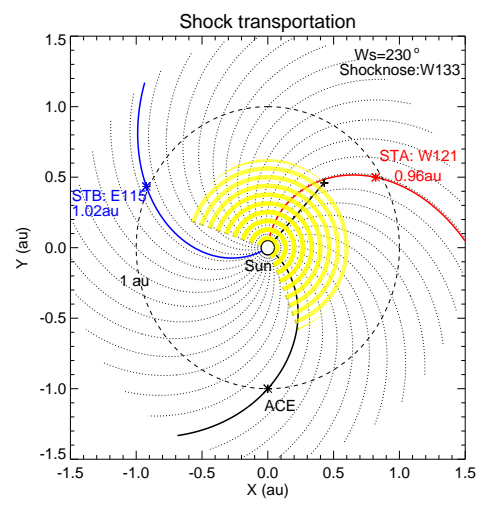

Figure 4: Same as Figure 1 except that the shock was at around 0.6 au on $T 1$, July 23, 14:08:00.

Around $T 1, A C E$ observed an SEP intensity maximum for protons, and after about 8 hours SEP intensity started to decrease (Figure 2). However, for electrons, around $T 1, A C E$ observed SEP intensity to continuously increase with time until it reached its maximum in a few hours and began to decrease after a few days (Figre 3). Figure 4 shows that at $T 1$ the shock arrived at the position around $0.63 \mathrm{au}$. We can see that the cobpoint of $A C E$ moved out from the edge of the shock, and so there was no connection between $A C E$ and the shock.

\section{Solar energetic particle transport model}

In this work, we use the shock particle transport code [3, 8] later abbreviated to SPTC [10] to simulate the transport of the 23 July 2012 SEP event assuming the CME-driven shock to be a moving particle source. For the study of the transport mechanism of the SEPs [11, 12], we use the three-dimensional focused transport equation [11-14]:

$$
\begin{array}{r}
\frac{\partial f}{\partial t}+\left(v \mu \hat{\mathbf{b}}+\mathbf{V}^{s w}\right) \cdot \nabla f-\nabla \cdot\left(\kappa_{\perp} \cdot \nabla f\right)-\frac{\partial}{\partial \mu}\left(D_{\mu \mu} \frac{\partial f}{\partial \mu}\right) \\
-p\left[\frac{1-\mu^{2}}{2}\left(\nabla \cdot \mathbf{V}^{s w}-\hat{\mathbf{b}} \hat{\mathbf{b}}: \nabla \boldsymbol{V}^{s w}\right)+\mu^{2} \hat{\mathbf{b}} \hat{\mathbf{b}}: \nabla \mathbf{V}^{s w}\right] \frac{\partial f}{\partial p} \\
+\frac{1-\mu^{2}}{2}\left[-\frac{v}{L}+\mu\left(\nabla \cdot \boldsymbol{V}^{s w}-3 \hat{\mathbf{b}} \hat{\mathbf{b}}: \nabla \mathbf{V}^{s w}\right)\right] \frac{\partial f}{\partial \mu}=0,
\end{array}
$$


where $D_{\mu \mu}$ is the particle pitch-angle diffusion coefficient following [15], [16], and [17]. A quasilinear model with the nonlinear effect of magnetic turbulence on the pitch angle scattering at $\mu=0$ [18] can be written as

$$
D_{\mu \mu}(\mu)=\frac{D_{\text {slab }} v}{l_{\text {slab }}}\left(\frac{R}{R_{a u}}\right)^{-1 / 3}\left(|\mu|^{g-1}+h\right)\left(1-\mu^{2}\right) .
$$

The relationship between the particle pitch angle diffusion coefficient and the parallel particle mean free path is $[15,19,20]$

$$
\lambda_{\|}=\frac{3}{8} v \int_{-1}^{1} \frac{\left(1-\mu^{2}\right)^{2}}{D_{\mu \mu}} \mathrm{d} \mu,
$$

and the parallel diffusion coefficient $\kappa_{\|}$can be written as $\kappa_{\|}=v \lambda_{\|} / 3$.

[21] developed nonlinear guiding center (NLGC) theory for perpendicular diffusion, which was approximated in analytic form [22] for particles in certain parameter and energy ranges,

$$
\kappa_{\perp}=\frac{1}{3} v D_{2 D} l_{2 D}^{2 / 3} \times \lambda_{\|}^{1 / 3}(\mathbf{I}-\hat{\boldsymbol{b}} \hat{\boldsymbol{b}})
$$

Here, $D_{2 D}$ is a parameter that depends on the spectral index in the inertial range and the $2 \mathrm{D}$ component of the magnetic turbulence.

Our model assumes the shock to be the source of particle injection with the boundary condition [23]:

$$
f_{b}=a \delta\left(r-v_{s} t\right)\left(\frac{r}{r_{c}}\right)^{\alpha} \exp \left[-\frac{|\phi(\theta, \varphi)|}{\phi_{c}(p)}\right] p^{\gamma} \xi(\theta, \varphi),
$$

where the shock acceleration efficiency parameter $\alpha$ measures the damping rate with radial distance, and the other shock acceleration strength parameter $\phi_{c}$ describes the injection decrease from the middle to the flank of the shock. Here, $\gamma$ is the power-law spectrum index of the shock, and $\xi(\theta, \phi)$ shows the angular range of the shock front [3],

$$
\xi(\theta, \varphi)= \begin{cases}1 & \text { if }|\phi(\theta, \varphi)| \leqslant \phi_{\mathrm{s}} \\ 0 & \text { otherwise }\end{cases}
$$

where $\phi(\theta, \varphi)$ is the angle between any particle injection position at the shock front and shock nose, and $\phi_{s}$ is the half angular width of the shock.

We reformulate the transport equation (1) in terms of a set of stochastic differential equations, and solve it with a time-backward Markov stochastic process method using the Monte Carlo simulation [24]. The particles are traced from the observation time back to the initial time of the injection at the source [11].

\section{Simulations and comparisons with observations}

From STEREO/SEPT we choose the proton channel with the energy range $2.2-6.5 \mathrm{MeV}$, and from ACE/EPAM we choose the proton channel with the energy range $1.91-4.75 \mathrm{MeV}$. We use 3 $\mathrm{MeV}$ as the typical value for simulations to compare with the three observational proton channels. 
For each simulated data point of energetic particle flux, $2.88 \times 10^{7}$ pseudo-particles are used. The observation and simulation results of the time profile of SEP fluxes are shown in Figure 5. The dotted and solid lines indicate observations and simulations, respectively. The black, red, and blue lines correspond to ACE, STEREO-A, and STEREO-B, respectively.

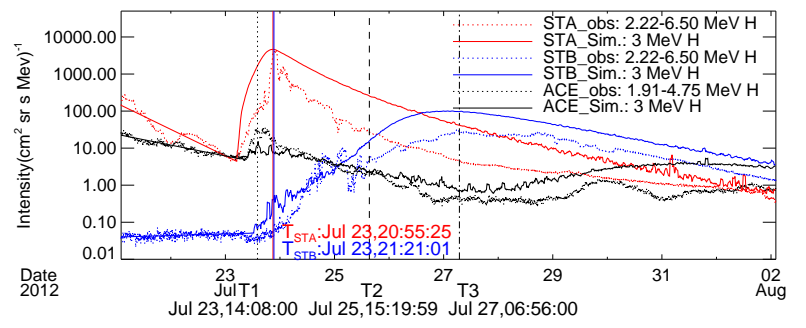

Figure 5: Proton fluxes of the observations and simulations during the 23 July 2012 SEP event.

From Figure 5 we can see that the simulations and observations generally agree well for the three spacecraft. Particularly in terms of the timing for the start and peak of SEP flux, the simulations approximately agree with observations.

We also perform numerical simulations for $0.2 \mathrm{MeV}$ electrons with $1.091 \times 10^{7}$ pseudo-particles for each data point. In Figure 6 we compare the simulation results with the electron observations of the EPAM instrument onboard ACE and the SEPT instruments onboard STEREO-A and STEREO-B. The ranges of energy we choose for the electron channel are $0.18-0.32 \mathrm{MeV}$ and $0.20-0.23$ $\mathrm{MeV}$ for EPAM and SEPT, respectively. From Figure 6 we can see that the observations and simulations of STEREO-A are consistent except that the peak of observations is about one order of magnitude higher. On the other hand, the STEREO-B observations, with a later starting time than simulations, have a peak that is lower than the simulated one. Furthermore, the $A C E$ observations and simulations agree relatively well. However, around $T 3$, there is an increase in the simulation of the flux as would be observed by ACE. In SEP events, reservoir phenomena are usually observed during which particle intensities are nearly the same at different locations in decay phase[e.g., 6-8].

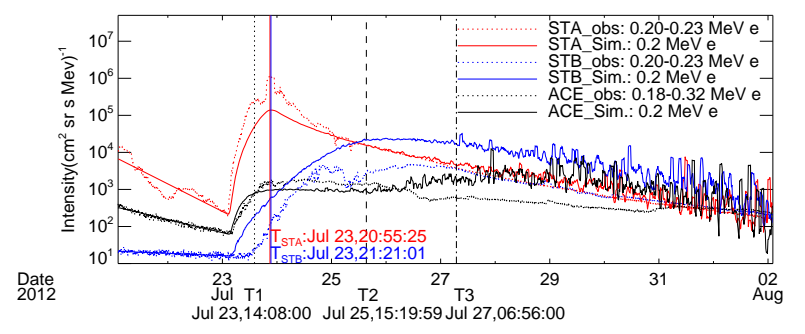

Figure 6: Electron fluxes of the observations and simulations during the 23 July 2012 SEP event.

\section{Conclusion and Discussion}

In this work, we investigate the 23 July 2012 SEP event observed by multi-spacecraft STEREO$A$, STEREO-B, and ACE. 
First, we used the Parker's spiral magnetic field model to qualitatively analyze the relationship between the propagation of the CME-driven shock in the interplanetary space and the associated SEP flux observed by the multiple spacecraft. We provide a schematic to show their relative positional relationship. Our analysis is able to qualitatively explain some of the important features, especially in terms of the timing for the start and peak of SEP flux observed by STEREO-A, STEREO-B, and $A C E$, simultaneously.

We then simulated the SEP event using the three-dimensional focused transport model, by treating the shock as a moving energetic particle source. In the simulations, almost all the important particle transport effects, such as solar wind convection, particle streaming along the magnetic field line, magnetic focusing, adiabatic cooling, and the diffusion coefficients parallel and perpendicular to the IMF, are included. The simulations and observations approximately agree for the three spacecraft, especially in terms of the timing for the start and peak of SEP flux. Further, the schematic can qualitatively describe some characteristics of simulations and observations.

\section{References}

[1] Y.D. Liu, J.G. Luhmann, P. Kajdič, E.K.J. Kilpua, N. Lugaz, N.V. Nitta et al., Observations of an extreme storm in interplanetary space caused by successive coronal mass ejections, Nature Communications 5 (2014) 3481 [1405 . 6088].

[2] C.T. Russell, R.A. Mewaldt, J.G. Luhmann, G.M. Mason, T.T. von Rosenvinge, C.M.S. Cohen et al., The Very Unusual Interplanetary Coronal Mass Ejection of 2012 July 23: A Blast Wave Mediated by Solar Energetic Particles, The Astrophysical Journal 770 (2013) 38 .

[3] Y. Wang, G. Qin and M. Zhang, Effects of Perpendicular Diffusion on Energetic Particles Accelerated by the Interplanetary Coronal Mass Ejection shock, .

[4] P. Riley, R.M. Caplan, J. Giacalone, D. Lario and Y. Liu, Properties of the Fast Forward Shock Driven by the July 232012 Extreme Coronal Mass Ejection, The Astrophysical Journal 819 (2016) 57 [1510.06088].

[5] A.M. Heras, B. Sanahuja, D. Lario, Z.K. Smith, T. Detman and M. Dryer, Three low-energy particle events: Modeling the influence of the parent interplanetary shock, The Astrophysical Journal 445 (1995) 497.

[6] R.B. McKibben, Azimuthal propagation of low-energy solar-flare protons as observed from spacecraft very widely separated in solar azimuth, Journal of Geophysical Research 77 (1972) 3957.

[7] E.C. Roelof, R.E. Gold, G.M. Simnett, S.J. Tappin, T.P. Armstrong and L.J. Lanzerotti, Low-energy solar electrons and ions observed at ULYSSES February-April, 1991 - The inner heliosphere as a particle reservoir, Geophysical Research Letters 19 (1992) 1243.

[8] G. Qin, Y. Wang, M. Zhang and S. Dalla, Transport of Solar Energetic Particles Accelerated by ICME Shocks: Reproducing the Reservoir Phenomenon, . 
[9] Y. Wang and G. Qin, Simulations of the Spatial and Temporal Invariance in the Spectra of Gradual Solar Energetic Particle Events, .

[10] S.Y. Qi, G. Qin and Y. Wang, Numerical simulations of solar energetic particle event timescales associated with icmes, Research in Astronomy and Astrophysics 809 (2017) 11.

[11] G. Qin, M. Zhang and J.R. Dwyer, Effect of adiabatic cooling on the fitted parallel mean free path of solar energetic particles, .

[12] M. Zhang, G. Qin and H. Rassoul, Propagation of Solar Energetic Particles in Three-Dimensional Interplanetary Magnetic Fields, .

[13] J. Skilling, Cosmic Rays in the Galaxy: Convection or Diffusion?, .

[14] R. Schlickeiser, Cosmic Ray Astrophysics.

[15] J.R. Jokipii, Cosmic-Ray Propagation. I. Charged Particles in a Random Magnetic Field, .

[16] A. Teufel and R. Schlickeiser, Analytic calculation of the parallel mean free path of heliospheric cosmic rays. II. Dynamical magnetic slab turbulence and random sweeping slab turbulence with finite wave power at small wavenumbers, .

[17] R.A. Burger, T.P.J. Krüger, M. Hitge and N.E. Engelbrecht, A Fisk-Parker Hybrid Heliospheric Magnetic Field with a Solar-Cycle Dependence, .

[18] G. Qin and A. Shalchi, Pitch-Angle Diffusion Coefficients of Charged Particles from Computer Simulations, .

[19] K. Hasselmann and G. Wibberenz, Scattering of Charged Particles by Random Electromagnetic Fields., .

[20] J.A. Earl, The diffusive idealization of charged-particle transport in random magnetic fields, .

[21] W.H. Matthaeus, G. Qin, J.W. Bieber and G.P. Zank, Nonlinear Collisionless Perpendicular Diffusion of Charged Particles, .

[22] A. Shalchi, G. Li and G.P. Zank, Analytic forms of the perpendicular cosmic ray diffusion coefficient for an arbitrary turbulence spectrum and applications on transport of Galactic protons and acceleration at interplanetary shocks, .

[23] M.-B. Kallenrode and G. Wibberenz, Propagation of particles injected from interplanetary shocks: A black box model and its consequences for acceleration theory and data interpretation, .

[24] M. Zhang, A Markov Stochastic Process Theory of Cosmic-Ray Modulation, .

[25] R.B. McKibben, J.J. Connell, C. Lopate, M. Zhang, A.D. Balogh, S., R.G. Marsden et al., ULYSSES COSPIN observations of the energy and charge dependence of the propagation of solar energetic particles to the Sun's south polar regions, International Cosmic Ray Conference 8 (2001) 3281. 